\title{
THE INFLUENCE OF THE ENVIRONMENT ON THE DYNAMIC SUSCEPTIBILITY OF CERIUM MAGNESIUM NITRATE IN STRONG MAGNETIC FIEI.DS
}

\author{
J.A. OVERWEG, J. FLOKSTRA and G.J. GERRITSMA \\ Twente University of Technology, P.O. Box 217, 7500 AE Enschede, The Netherlands
}

Received 1 September 1981

\begin{abstract}
Dynamic susceptibility curves observed as a function of frequency often deviate considerably from curves predicted by the simple Casimir-Du Pré model. In the case of cerium magnesium nitrate these deviations are mainly caused by the poor thermal conductivity of the liquid or gaseous helium that surrounds the sample and becomes extremely large when the external magnetic field becomes larger than approximately $1 \mathrm{~T}$. We found that, even in this extreme situation, the influence of the environment can be accurately described with the thermal conduction model developed by Flokstra et al.

We also discovered that the lattice temperature is stabilized much more effectively if the sample is placed in helium gas at its saturation pressure. The thin helium film that is formed on the surface of the sample provides, through condensation and reevaporation, a far better thermal stabilization of the lattice than bulk liquid helium does. The susceptibility curves that are observed under these conditions differ much less from the Casimir-Du Pré curves.
\end{abstract}

\section{Introduction}

A commonly used technique for studying the magnetic properties of materials is to measure the changes in magnetization of a sample due to a harmonic external magnetic field. A series of such dynamic susceptibility measurements as a function of the frequency can be used to determine the spin-lattice relaxation time of the substance. This time constant is usually derived from the frequency $f^{*}$ for which the imaginary component $\chi^{\prime \prime}$ of the complex susceptibility has a maximum $\left(\tau=1 / 2 \pi f^{*}\right)$. This method works as long as the system under investigation behaves according to the Casimir-Du Pré model. However, observed susceptibility curves often deviate from the shape predicted by this simple model. Sometimes, these deviations are caused by intrinsic properties of the material. Flokstra et al. [1,2] have shown that under certain conditions large deviations can be caused by the limited thermal conduction in the liquid or gaseous helium environment of the sample. Cal- culations with their thermal conduction model, in which these effects are taken into account, are in good agreement with observed dynamic susceptibility curves.

The thermal conduction model has so far only been tested quantitatively on systems where the environmental influence is moderate. The present paper describes experiments on cerium magnesium nitrate $(\mathrm{CMN})$ where the influence of the environment can become quite large (section 2). We then found that the thermal conduction model still gives an excellent description of the observed susceptibility curves (section 4).

In the course of our experiments on CMN we also discovered that almost ideal susceptibility curves are obtained, when the sample is placed in helium gas at its saturation pressure instead of in liquid helium. This improvement is caused by the thin helium film which is then adsorbed at the surface of the sample. The temperature of the sample is effectively stabilized by periodic condensation and evaporation of helium at the surface of this film and thus the Casimir-Du Pré 
conditions are much more closely fulfilled than when the sample is surrounded by liquid or gaseous helium (sections 5-7).

\section{Field and temperature range where a large environmental influence can be expected}

The exact formulae for the susceptibility (dependent on the particular crystal geometry) are rather complicated $[1,2]$. They can be greatly simplified when the thermal conductivity $\lambda_{L}$ of the sample is much larger than that of the surroundings $\left(\lambda_{g}\right)$ and when also the specific heat $C_{H}$ of the spin system is larger than the lattice specific heat $C_{\mathrm{L}}$. The simplified model predicts a large environmental influence when a single parameter $E$ is much larger than unity. For a spherical sample, $E$ is equal to [3]

$E=\frac{C_{H} \alpha}{C_{\mathrm{g}} \lambda_{\mathrm{g}} / r^{2}}$

where $\alpha$ is the heat transfer coefficient $C_{H} / \tau$ between the spin system and the lattice and $r$ is the radius of the sample.

Cerium magnesium nitrate $\left(\mathrm{Ce}_{2} \mathrm{Mg}_{3}\left(\mathrm{NO}_{3}\right)_{12}\right.$. $24 \mathrm{H}_{2} \mathrm{O}$ ) has been investigated extensively in the past and its relaxation behaviour is well understood. The bottlenecked Orbach process is the dominating relaxation mechanism in a large field and temperature region and the relaxation time obeys $[4,5]$

$\tau=A H^{1 / 2} \mathrm{e}^{36.25 / T}$

where $A$ is in the order of $10^{-12} \mathrm{~s} \mathrm{~m}^{1 / 2} \mathrm{~A}^{-1 / 2}$. The spin specific heat can be calculated with [6]

$$
C_{H}=\mu_{0} C H^{2} / T^{2}
$$

The Curie constant $C$ of $\mathrm{CMN}$ with $H$ perpendicular to the $c$ axis is $C_{\perp}=0.98 \times 10^{-2} \mathrm{~K}$. The lattice specific heat is given by Colwell [7].

Fig. 1 presents calculated values of the

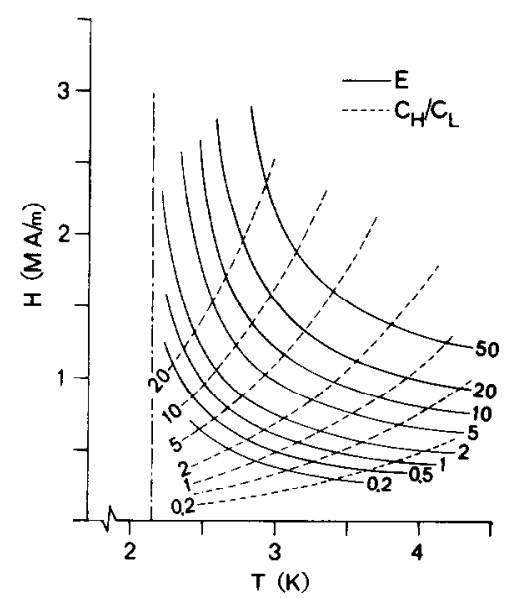

Fig. 1. Contours of constant $E$ and $C_{H} / C_{\mathrm{L}}$.

environment parameter $E$ and of the specific heat ratio $C_{H} / C_{\mathrm{L}}$ as a function of $H$ and $T$ for a spherical sample of $2 \mathrm{~mm}$ radius using for $C_{\mathrm{g}}$ and $\lambda_{\mathrm{g}}$ the values for liquid helium. The figure shows that a large field and temperature region exists where both $E$ and $C_{H} / C_{\mathrm{L}}$ are large with respect to unity. Near $4.2 \mathrm{~K}$ and in magnetic fields above approximately $1 \mathrm{~T}, E$ can become as large as 50 . Under these conditions a very large influence of the environment upon the observed dynamic susceptibility can be expected.

The thermal conduction model has so far been tested on substances like potassium chromium alum [2] which show only a moderate environmental influence ( $E$ is in the order of unity). By performing dynamic susceptibility measurements on $\mathrm{CMN}$ in strong magnetic fields we can test the applicability of the thermal conduction model in an extreme situation.

\section{Experimental set-up}

The dynamic susceptibility measurements were carried out with a conventional mutual inductance bridge technique [8]. After compensation of the imbalance and the losses of the empty sensing mutual inductor, the off-balance signal resulting from the insertion of the sample 
into the sensing coils was taken as a measure for the susceptibility of the sample. A magnetic field of up to $2 \mathrm{~T}$ was generated by a superconducting solenoid.

The sample was separated from the liquid helium, in which the coil system was immersed, by a quartz tube connected to a pressure control system outside the cryostat. The magnetic properties of the sample could thus be studied under various environmental conditions from complete thermal insulation (helium pressure $p<$ $10^{-6}$ Torr) to good thermal contact with gaseous or liquid helium.

\section{Observed and calculated dynamic suscep- tibility of CMN}

At two different temperatures, 4.2 and $3.5 \mathrm{~K}$, and in a constant magnetic field of $2 \mathrm{~T}(H=$ $1.6 \mathrm{MA} / \mathrm{m}=20 \mathrm{kOe}$ ) we measured the dynamic susceptibility of a CMN sample as a function of the frequency, with the sample in liquid helium, in vacuum and in helium gas near its saturation pressure. The sample was a single crystal (size $10 \times 2 \times 3 \mathrm{~mm}^{3}$ ), mounted with its $c$ axis perpendicular to the magnetic field. Fig. 2 shows the results of these two sets of experiments. As could be expected from the foregoing considerations the shapes of the observed 'liquid-helium' susceptibility curves ( $\Delta$ in fig. 2 ) deviate strongly from those predicted by the Casimir-Du Pré model. Especially at $4.2 \mathrm{~K}$ it seems impossible to derive the value for the intrinsic spin-lattice relaxation time from these data. This relaxation time was determined from the measurements in vacuum ( $\square$ in fig. 2). If the sample is thermally isolated from its surroundings the relaxation time that follows from the maximum of the vacuum absorption curve is related to $\tau_{\mathrm{sL}}$ by [9]:

$\tau_{\mathrm{vac}}=\tau_{\mathrm{sL}} C_{\mathrm{L}} /\left(C_{H}+C_{\mathrm{L}}\right)$

The relaxation times thus obtained are in good agreement with eq. (2). The Casimir-Du Pré curves that correspond to these relaxation times are also included in fig. 2 (dotted curves). These curves would have been found if the thermal conductivity of the liquid helium had been infinite.

The influence of the liquid-helium environment has been calculated with the complete thermal conduction model [1]. The results of these calculations, which are included in fig. 2 (solid lines
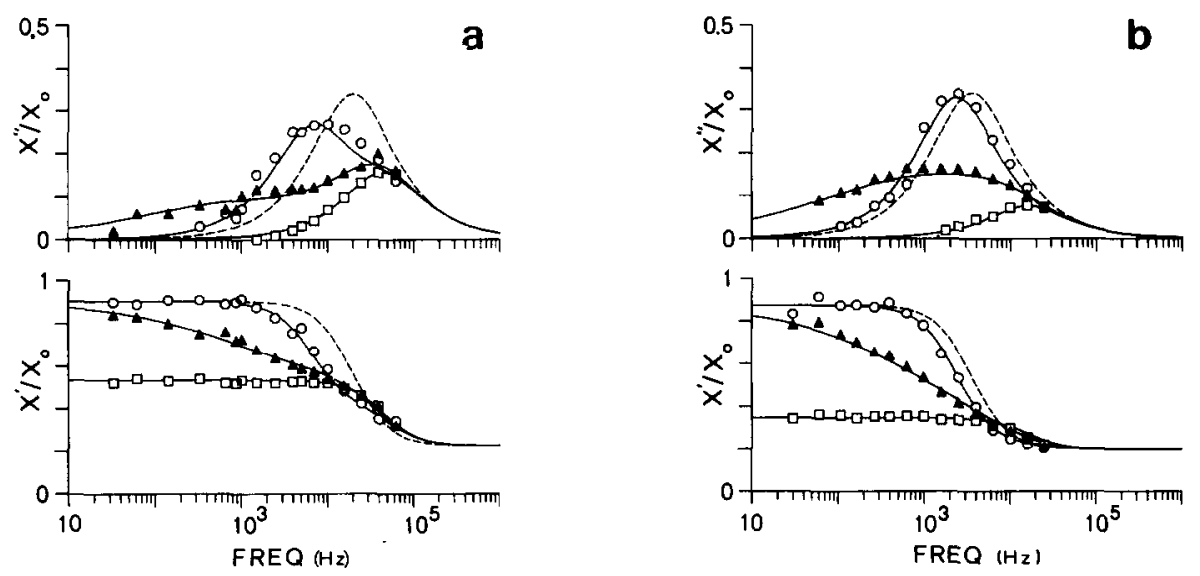

Fig. 2. Dynamic susceptibility of CMN vs. frequency. Experimental conditions: $H=1.5 \mathrm{MA} / \mathrm{m}$; (a) $T=4.2 \mathrm{~K}$, (b) $T=3.5 \mathrm{~K}$; $\Delta$ liquid helium, $\square$ vacuum, $O$ saturated vapour. Solid lines represent calculations corresponding to data points. The dotted curves would be observed in the case of ideal thermal contract between the sample and its surroundings (Casimir-Du Pré model). $\chi_{0}=\chi_{T}(H=0)$. 
through $\Delta$ ), are in very good agreement with the measurements. We may therefore conclude that even in this extreme situation the thermal conduction model gives an accurate description of the observed dynamic susceptibility curves.

Because of the excellent agreement between the experimental data and the calculated susceptibility curves we could, with reasonable confidence, calculate the form of the susceptibility curves at other temperatures and fields. Typical examples of calculated $\chi^{\prime \prime}$ vs. $\chi^{\prime}$ curves at 4.2 and $2.7 \mathrm{~K}$ are shown in fig. 3. The magnetic field increases from 0.15 to $3.0 \mathrm{MA} / \mathrm{m}$, in steps of $0.15 \mathrm{MA} / \mathrm{m}$. In this field and temperature range the bottlenecked Orbach process (eq. (2)) is effective. Because of the very small internal field the high-frequency limit of the susceptibility can be taken as equal to the Van Vleck temperatureindependent susceptibility.

At weak fields there are no significant deviations from the semicircles predicted by the Casimir-Du Pré model. At higher values of the external field the curves become flattened due to
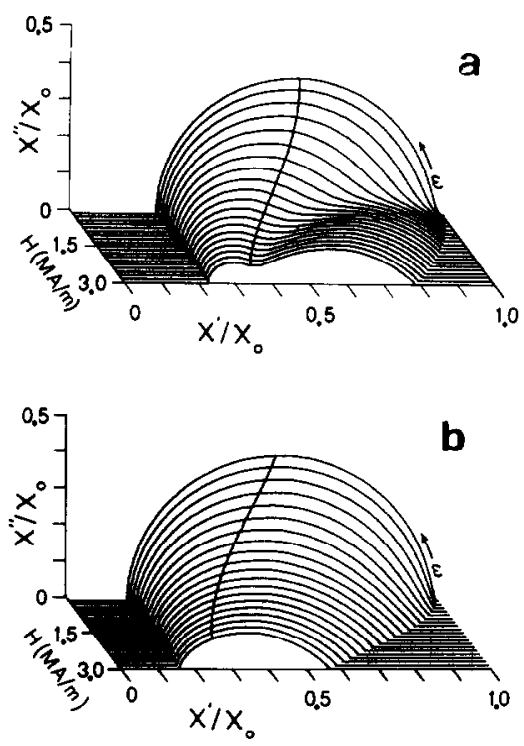

Fig. 3. Calculated curves of $\chi^{\prime \prime}$ vs. $\chi^{\prime}$ for CMN liquid helium, plotted at $0.15 \mathrm{MA} / \mathrm{m}(0.2 \mathrm{~T})$ intervals. (a) $T=4.2 \mathrm{~K}$, (b) $T=2.7 \mathrm{~K}$. Also included is the locus of points for which $\omega=1 / \tau_{\text {sL. }}$. the lattice-to-bath relaxation and at $4.2 \mathrm{~K}$ the curves even split up into two parts. The part on the left represents the relaxation from the spins to the isolated lattice while the other part corresponds to the lattice-to-bath relaxation. The points for which the frequency corresponds to the inverse spin-lattice relaxation time are also shown in fig. 3. In strong magnetic fields the spin-lattice relaxation time constant can obviously not be taken in the usual manner from the maximum of $\chi^{\prime \prime}$. Under these conditions a reliable value for the spin-lattice relaxation time can, in principle, be obtained from vacuum measurements using eq. (4).

\section{Experiments in helium gas at saturation pres- sure}

If a CMN sample is surrounded by gaseous helium and its dynamic susceptibility is recorded as a function of time while the pressure of the gas is very slowly increased, an interesting effect is observed when the pressure approaches its saturation value $p_{0}$. Fig. 4 shows a typical example of such a recording $(T=3.5 \mathrm{~K}, H=$ $1.5 \mathrm{MA} / \mathrm{m}$, frequency $630 \mathrm{~Hz}$ ). the susceptibility increases slightly (A-B) until the pressure is approximately 2 Torr below its saturation value (C). Then the dispersion $\chi^{\prime}$ increases at a much faster rate and after a few minutes it stabilizes at

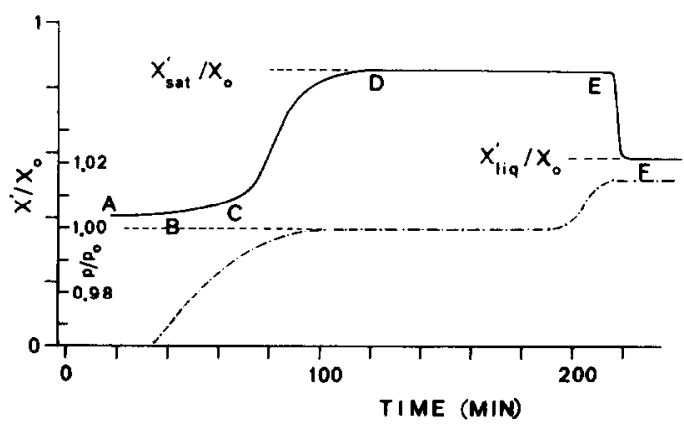

Fig. 4. Example of recording of susceptibility during formation of helium film on sample surface (for A-F: see text). CMN, $T=3.5 \mathrm{~K}, \quad H=1.5 \mathrm{MA} / \mathrm{m}$, frequency: $630 \mathrm{~Hz}$ $\left(1 / 2 \pi \tau_{\mathrm{lL}}=3500 \mathrm{~Hz}\right)$. 
an equilibrium value (D-E) which will be referred to as the saturation value. When the pressure of the helium gas in the sample tube is held just above the saturation pressure for a sufficiently long time, the susceptibility suddenly changes to another equilibrium value $(F)$. This change occurs when the level of the liquid, formed by condensation in the sample tube, reaches the crystal and the final susceptibility is just the value found in the previously described 'liquidhelium' experiments. similar effects are observed in $\chi^{\prime \prime}$.

The intermediate (D-E) saturation value is less easily explained. Measurements of the saturation value $\chi_{\text {sat }}^{\prime}$ as a function of the frequency are included in fig. 2. From this figure it is clear that at saturated vapour pressure, experiments yield susceptibility curves which deviate much less from the ideal ones than the 'liquid-helium' curves do. When a sample is placed in helium gas at saturation pressure the heat exchange with its surroundings is apparently much better than when it is immersed in liquid helium.

A qualitative explanation of this effect is rather simple. In saturated helium gas the surface of the sample will be covered with a thin liquid-helium film. Any energy supplied to the crystal by a small increase of the external magnetic field will be removed immediately by partial evaporation of this film. Similarly any withdrawal of energy will be compensated by condensation of more helium. Because the surface of the adsorbed film behaves isothermally the amplitude of the oscillations in the lattice temperature is much smaller than if the sample were immersed in a bath of liquid helium. The Casimir-Du Pré conditions will thus be more closely fulfilled, resulting in more ideal susceptibility curves.

\section{Some remarks on the dynamics of the for- mation of the helium film}

When during the formation of the helium film on the surface of the sample the pressure is increased slowly (at a rate of about $0.1 \mathrm{Torr} / \mathrm{min}$ or less) the observed susceptibility will depend only on the pressure of the helium gas with respect to $p_{0}$ (as shown in fig. 4). When the pressure is changed at a much faster rate (e.g. 1 Torr $/ \mathrm{min}$ ), several remarkable effects are observed. After an increase of the pressure the susceptibility approaches exponentially towards a new equilibrium value, as illustrated in fig. 5 , but usually there is first a slight drop in $\chi^{\prime}$ (A-B and E-F). The response to a small decrease of the pressure $\left(0.002 p_{0}\right.$ is sufficient) is even more peculiar (C, G, I, J). The susceptibility immediately jumps to the saturation value and after a few seconds, depending on the amount of helium that was adsorbed before the pressure change, a second jump occurs to the susceptibility corresponding to the final pressure $(\mathrm{D}, \mathrm{H}, \mathrm{I}, \mathrm{J})$. Apparently it is not only the actual thickness of the film but also the external pressure that determine the thermal properties of the film. We found that the strange dynamic effects described above are no longer observed when the temperature is lowered below the superfluid transition temperature $T_{\lambda}$ of liquid helium. Instead, the susceptibility then immediately changes to the value corresponding to the new pressure. Under quasi-stationary conditions, however, the behaviour of $\chi$ as a function of the

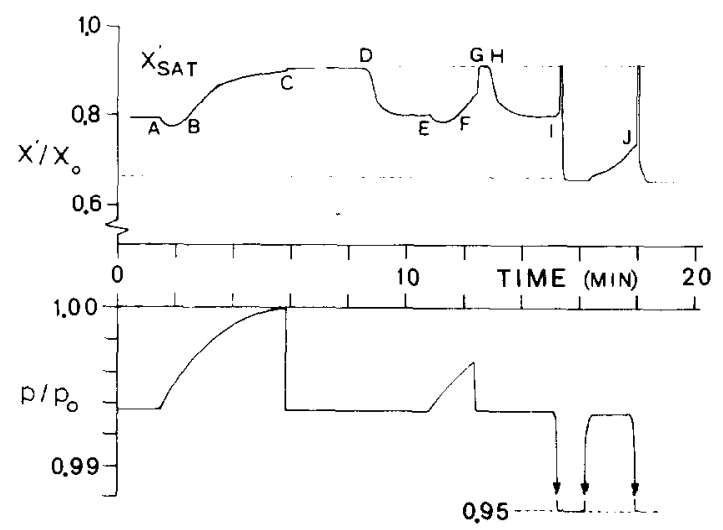

Fig. 5. Compilation of effects encountered during experiments in helium at saturation pressure (for A-J: see text). $\mathrm{CMN}, T=4.2 \mathrm{~K}, H=1.5 \mathrm{MA} / \mathrm{m}$, frequency: $620 \mathrm{~Hz}$. 
helium pressure is analogous to that observed above $T_{\lambda}$ (fig. 4).

\section{Further analysis of the effect of the adsorbed helium film}

There remain two questions, that need some further investigation:

(i) The nearly ideal susceptibility curves are not observed when the pressure of the gas is not very close to $p_{0}$. Why is this so?

(ii) The susceptibility curves observed at saturation pressure still deviate slightly from the ideal ones (especially near $4.2 \mathrm{~K}$ ). What is the nature of the remaining small thermal resistance in this situation?

\subsection{Pressure dependence of the observed suscep- tibility}

If the heat transfer between the crystal and its surroundings only takes place through ordinary thermal conduction in the helium gas the observed susceptibility curves will deviate from the ideal ones in much the same way as when the crystal is immersed in a bath of liquid helium. More ideal curves like those observed near $p_{0}$ can only be obtained if there is an additional heat transfer mechanism effective, such as that described in previous sections. The fact that Casimir-Du Pré-like susceptibility curves are not observed below $p_{0}$, implies that either the efficient heat transfer through evaporation and condensation does not take place in this situation, or if it does, there is a thermal resistance between the bulk of the crystal and the surface of the adsorbed film, which is large at pressures below $p_{0}$.

The crystal will already be covered with a helium film of a few atomic layers thickness at pressures far below the saturation pressure [10]. As the thermal properties of such an unsaturated film (e.g. the heat of evaporation) are reported to be almost equal to those of bulk liquid helium one might expect that the proposed helium film effect could well be effective at pressures far below $p_{0}$.

The condensation-evaporation process would obviously not be possible if the heat fluxes to and from the crystal due to the oscillating magnetic field are so large that the changes in film thickness would be larger than the actual thickness of an unsaturated film. The following simple calculation shows that this is not the case under our measuring conditions.

Suppose that the heat, supplied to a spherical crystal of radius $r$ by an increase of the external magnetic field $H$ by an amount $\mathrm{d} H$, is completely absorbed by the evaporation of a layer of thickness $\delta$ from the surface of the film. Then the heat balance for this process is in first approximation equal to

${ }_{3}^{4} \pi r^{3} \mu_{0}(C / T) H \mathrm{~d} H=r \pi r^{2} L \delta$,

so that

$\delta=\mu_{0} r(C / T) H \mathrm{~d} H / 3 L$,

where $L$ is the heat of evaporation of the helium, which we assume to be equal to that of bulk liquid: $L=2.6 \times 10^{6} \mathrm{~J} / \mathrm{m}^{3}$. In our case the resulting fluctuations in the film thickness are in the order of $10^{-10} \mathrm{~m}$ so they can be safely neglected. Obviously, there must be another reason why the condensation-evaporation mechanism is no longer effective at pressure below $p_{0}$.

The evaporation and condensation processes at the surface of the film are of course never perfectly isothermal. The temperature drop across the liquid-vapour interface is characterized by the Knudsen resistance $\boldsymbol{R}_{\mathrm{Kn}}$ [11]. We calculated, however, that $R_{\mathrm{Kn}}$ is so small, that it cannot cause the large changes observed in $\chi$ below $p_{0}$. Apart from this its pressure dependence is not strong enough to explain the observed rapid change of $\chi$ in a very small pressure interval near $p_{0}$.

As a next step we will assume that the surface 
of the helium film can be considered isothermal and we shall look for a pressure-dependent thermal resistance, between the sample and the surface of this film, which could explain the observed deviations of the susceptibility curves. Using the thermal conduction model, we calculated that this resistance has to be at least in the order of $10^{-5} \mathrm{~m}^{2} \mathrm{~K} / \mathrm{W}$ in order to cause any noticeable effect and more than $10^{-4} \mathrm{~m}^{2} \mathrm{~K} / \mathrm{W}$ to explain the large deviations we observed below $p_{0}$.

The bulk of the helium film will act as a small thermal resistance between the crystal surface and the liquid-vapour interface, but its effect is too small. In order to cause any change in the susceptibility the thickness of the film would have to be at least $10^{-7} \mathrm{~m}$ or about 200 atomic layers. Helium films of such a thickness have only been observed below the lambda point of liquid helium [12]. Otherwise the effect of the resistance would become larger when the pressure approaches $p_{0}$, which is in contrast to the experimental observations.

Then there is the Kapitza resistance, which governs the heat transfer at the solid-liquid boundary. Although this resistance has some influence at the saturation pressure (see section 7.2), its effect on the susceptibility is too small to explain our results below $p_{0}$. Furthermore, it is not likely that the Kapitza resistance would be sensitive to changes in the pressure of the helium gas.

Thus, although our experiments indicate that heat transfer through evaporation and condensation is only effective at saturation pressure, we cannot find a proper explanation for this fact. Maybe the structure of the surface of the crystal has to be taken into account. For aqueous crystals however, the surface is not well defined and this makes a further analysis difficult.

\subsection{Remaining thermal resistance at saturation pressure}

In the final equilibrium situation, where the thickness of the adsorbed film may be more than 50 atomic layers, the heat transfer between the surface of the film and the vapour phase can be considered ideal. As was already mentioned in section 7.1 the remaining deviation cannot be caused by the thermal resistance of the bulk of the film. The only other thermal resistance that could influence the relaxation process is the Kapitza resistance $\boldsymbol{R}_{\mathrm{K}}$. We calculated the influence of a thermal resistance of the form $K T^{-3}$ between the sample and a perfect heat reservoir. A reasonable agreement with our 'saturated vapour' curves was obtained when $R_{\mathrm{K}}=7 \times 10^{-4} \mathrm{~T}^{-3}\left(\mathrm{~m}^{2} \mathrm{~K} / \mathrm{W}\right)$. The results of these calculations are included in fig. 2 (solid lines through $O$ ). The value for $R_{K}$ thus obtained is in good agreement with the results of Glättli [13] and Groenendijk et al. [14]. Glättli found that the Kapitza resistance between cerium ethyl sulphate and superfluid helium obeys the relation: $R_{\mathrm{K}}=3 \times 10^{-3} \mathrm{~T}^{-2.4}\left(\mathrm{~m}^{2} \mathrm{~K} / \mathrm{W}\right)$. Groenendijk et al, determined the Kapitza resistance from relaxation measurements in the magnetic ordered phase of $\mathrm{MnCl}_{2} \cdot 4 \mathrm{H}_{2} \mathrm{O}$ and $\mathrm{MnBr}_{2} \cdot 4 \mathrm{H}_{2} \mathrm{O}$ and found $1.9 \times 10^{-3} T^{-3}\left(\mathrm{~m}^{2} \mathrm{~K} / \mathrm{W}\right)$ and $0.89 \times 10^{-3} T^{-3}\left(\mathrm{~m}^{2}\right.$ $\mathrm{K} / \mathrm{W}$ ), respectively.

Note that, although the Kapitza resistance decreases rapidly at higher temperatures, the effect of this resistance is largest, in our measurements, at $4.2 \mathrm{~K}$. This is because of the even more rapid (exponential) decrease of the spin-lattice relaxation time of the bottlenecked Orbach process with temperature.

\section{Conclustons}

The spin-lattice relaxation of $\mathrm{CMN}$ is, in strong magnetic fields, severely influenced by the thermal properties of the surroundings of the sample. These thermal conduction effects can be minimized by placing the sample in helium gas at the saturation pressure. The helium film formed on the surface of the sample provides an effective thermal stabilization of the crystal. Under these conditions the only thermal resis- 
tance that influences the relaxation process is the Kapitza resistance between the surface of the crystal and the helium film.

\section{Acknowledgements}

We wish to thank Prof. Dr. L.C. van der Marel for many stimulating and helpful discussions and Dr. A.J. van Duyneveldt for critically reading the manuscript.

\section{References}

[1] J. Flokstra, G.J. Gerritsma, G.A. Hartemink and L.C. van der Marel, Physica 77 (1974) 99.

[2] G.J. Gerritsma, J. Flokstra, G.A. Hartemink, J.J.M. Scholten, A.J.W.A. Vermeulen and L.C. van der Marel, Physica 95B (1978) 173.
[3] The expression for $E$ given here was derived from the general formulae of the heat conduction model given in ref. [1], assuming that the influence of the boundary resistance can be neglected.

[4] A. van der Bilt and A.J. van Duyneveldt, J. de Physique C6 (1978) 1002.

[5] J.T. Hoffman and R.C. Sapp, J. Appl. Phys. 39 (1968) 837.

[6] W.F. Giauque, R.A. Fisher, E.W. Hornung and G.E. Brodale, J. Chem. Phys. 58 (1973) 2621.

[7] J.H. Colwell, J. Low Temp. Phys. 14 (1974) 53.

[8] J. Flokstra, G.J. Gerritsma, H.J.M. Kreuwel and L.C. van der Marel, J. Phys. E13 (1980) 1071.

[9] J. Flokstra, G.J. Gerritsma and L.C. van der Marel, Physica 94B (1978) 53.

[10] See e.g. E. Long and L. Meyer, Adv. Phys. 2 (1953) 1.

[11] E.H. Kennard, Kinetic Theory of Gases (McGraw-Hill, New York, 1938) p. 314.

[12] L.G. Grimes and L.C. Jackson, Phil. Mag. 50 (1959) 1346.

[13] H. Glättli, Can. J. Phys. 46 (1968) 103.

[14] H.A. Groenendijk, J. Flokstra and A.J. van Duyneveldt, Physica 112B (1982) 33. 